\title{
Covid-19 Vaccine Authorized in India-A Mini Review
}

\author{
Dr. Yogendra Shrestha ${ }^{1}$, Rajesh Venkatraman ${ }^{1}$, Jeet Bahadur Moktan ${ }^{2}$, Renukaradhya \\ chitti $^{2}$, and Shiv Kumar Yadav ${ }^{2}$ \\ ${ }^{1}$ Adichunchanagiri University \\ ${ }^{2}$ Affiliation not available
}

May 6, 2021

\begin{abstract}
Background: Many variants detected after Wuhan-Hu-1 reference which were able to develop the resistance against the neutralizing antibodies induced by vaccine and may cause false negative results in diagnostic tests targeting the $\mathrm{S}$ gene. The Covid-19 cases are increasing rapidly in India and the government authorized a new vaccine for restricted use in emergency situation. Aims: This review discusses about the efficacy, safety, and economical aspects of vaccines authorized in India. Materials and methods: Wide-ranging assessment and analysis of accessible resources on online database. Results: The rAd26-s \& rAd5-s (Gamaleya Research Institute of Epidemiology and Microbiology) demonstrate high efficacy as well as safety, followed by BBV152 (Bharat Biotech) and AZD1222 (Oxford AstraZeneca); AZD1222 is more economical than other two. Conclusion: The main goal of vaccination is to reduce deaths, protect the health care system and finally disease control, this is only be possible if more population is vaccinated with locally available Covid-19 vaccine and self-awareness about the prevention of disease transmission. Key Words: Covid-19, SARS-CoV-2, Immunization, Vaccine, Efficacy.
\end{abstract}

\section{Hosted file}

IJCP.pdf available at https://authorea.com/users/412175/articles/520946-covid-19-vaccineauthorized-in-india-a-mini-review 